\title{
Lipid Abnormalities During Acute Metabolic Complications of Diabetes in Japanese Diabetic Patients
}

\author{
Hidekatsu Yanaia, b, Sumie Moriyama $^{\mathrm{a}}$
}

\section{To the Editor}

Severe hypertriglyceridemia and low high-density lipoprotein cholesterol (HDL-C) were observed in patients with diabetic ketosis (DK) and diabetic ketoacidosis (DKA) [1]. However, lipid abnormalities during acute metabolic complications of diabetes such as DKA, DK and hyperglycemic hyperosmolar syndrome (HHS) remain largely unknown.

We retrospectively studied serum lipid profiles in patients with DKA $(n=7)$, DK $(n=24)$ and HHS $(n=9)$. The study protocol was approved by the Ethics Committee of the $\mathrm{Na}$ tional Center for Global Health and Medicine, and the study was performed in accordance with the Declaration of Helsinki. The mean \pm standard deviation (SD) of age, body height, body weight, body mass index (BMI), HbAlc in DKA, DK and HHS were $49.1 \pm 9.2,51.9 \pm 14.1$ and $60.4 \pm 16.5$ years, 167.2 $\pm 10.9,166.8 \pm 8.8$ and $159.2 \pm 8.3 \mathrm{~cm}, 71.1 \pm 19.0,70.7 \pm$ 21.1 and $62.5 \pm 7.2 \mathrm{~kg}, 25.0 \pm 4.7,25.3 \pm 6.6$ and $24.7 \pm 2.3 \mathrm{~kg} /$ $\mathrm{m}^{2}, 9.6 \pm 4.9,11.3 \pm 4.2$ and $10.8 \pm 3.0 \%$, respectively.

Serum triglyceride (TG) and HDL-C, plasma glucose (PG) and blood $\mathrm{pH}$ in DKA, DK and HHS were shown in Figure 1. Serum TG in patients with DKA which is induced by severe insulin deficiency was the highest, and TG in HHS which commonly occurs in type 2 diabetes was the lowest. HDL-C in DKA was lower than that in DK. HDL-C was lower in the order of HHS, DKA, DK.

To elucidate what induces these lipid abnormalities, we studied correlation of TG, HDL-C with other metabolic parameters. Age, BMI and $\mathrm{HbAlc}$ were not correlated with either TG or HDL-C. PG was significantly and positively correlated with serum TG $(\mathrm{r}=0.496, \mathrm{P}=0.001$, by Pearson's correlation $)$. However, PG was not correlated with HDL-C. Blood $\mathrm{pH}$ reflects ketone body production by insulin deficiency, and blood $\mathrm{pH}$ was significantly and negatively correlated with serum TG $(\mathrm{r}=-0.533, \mathrm{P}<0.001)$ (Fig. 2), suggesting that severe insulin deficiency induced hypertriglyceridemia. However, blood $\mathrm{pH}$

Manuscript submitted June 6, 2019, accepted June 11, 2019

aDepartment of Internal Medicine, National Center for Global Health and Medicine, Kohnodai Hospital, Chiba, Japan

${ }^{b}$ Corresponding Author: Hidekatsu Yanai, Department of Internal Medicine, National Center for Global Health and Medicine, Kohnodai Hospital, 1-7-1 Kohnodai, Chiba 272-8516, Japan. Email: dyanai@hospk.ncgm.go.jp

doi: https://doi.org/10.14740/jem586 was not correlated with serum HDL-C. In DKA/DK patients, serum TG was significantly and positively correlated with PG $(\mathrm{r}=0.417, \mathrm{P}=0.007)$, and tended to be negatively correlated with blood $\mathrm{pH}(\mathrm{r}=-0.376, \mathrm{P}=0.053)$, suggesting a significant association between hypertriglyceridemia and insulin deficiency. In HHS patients, serum TG was significantly and positively correlated with $\mathrm{PG}(\mathrm{r}=0.842, \mathrm{P}=0.004)$; however, was not correlated with blood $\mathrm{pH}$. Because insulin deficiency in HHS is milder than that in DKA and DK, hyperglycemia, not blood $\mathrm{pH}$, may reflect insulin deficiency. Serum HDL-C was not correlated with $\mathrm{PG}$ and blood $\mathrm{pH}$ in both DKA/DK and HHS patients.

The DK/DKA patients have been reported to show a decrease in lipoprotein lipase (LPL), the rate-limiting enzyme for hydrolysis of TG in TG-rich lipoproteins such as chylomicron (CM), intermediate-density lipoprotein (IDL) and very lowdensity lipoprotein (VLDL), which, after insulin therapy, was normalized with a concomitant normalization in serum TG [2]. The formation of HDL has been reported to be related to the LPL-mediated catabolism of TG-rich lipoproteins other than

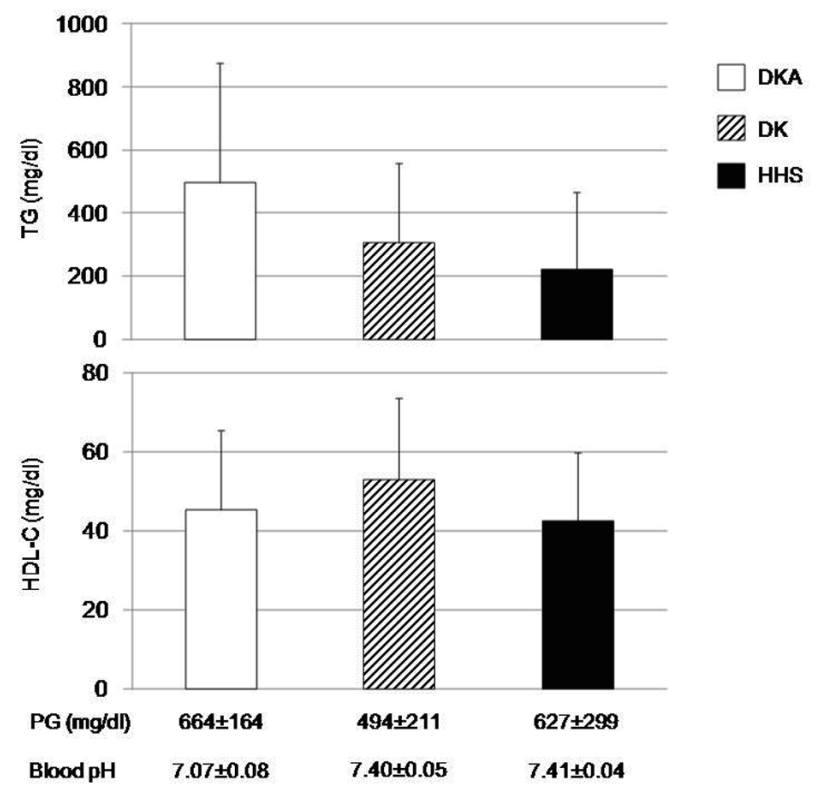

Figure 1. Serum TG, $P G$ and blood $p H$ in patients with DKA, DK and HHS. Bars and error bars indicate mean and SD, respectively. TG: serum triglyceride; PG: plasma glucose; DKA: diabetic ketoacidosis; DK: diabetic ketosis; HHS: hyperglycemic hyperosmolar syndrome. 


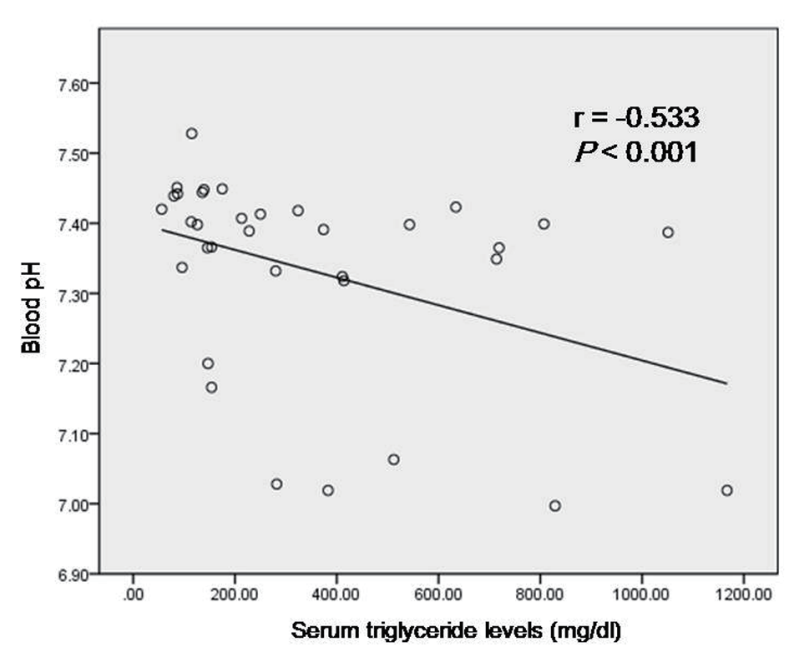

Figure 2. Correlation between serum triglyceride levels and blood pH.

CM such as IDL and VLDL [3]. Serum TG was not correlated with serum HDL-C, and HDL-C was not associated with insulin-deficient state such as blood $\mathrm{pH}$ and $\mathrm{PG}$ in present study. Under insulin-deficient state, VLDL may not be produced because free fatty acids as the material for VLDL become ketone bodies. During acute metabolic complications of diabetes, elevated CM may be the main cause of hypertriglyceridemia.

We have to mention the limitation of present study. The number of subjects was small. To elucidate our hypothesis, further studies, preferably with larger numbers of subjects, will be needed.

In conclusion, during acute metabolic complications of diabetes such as DKA, DK and HHS, hypertriglyceridemia may be induced by insulin deficiency.

\section{Acknowledgments}

We thank the staff (Yukie Kawamura, Keiko Nakamura, Harue Aoki and Ayano Sakakibara) of the Division of Research Sup- port, National Center for Global Health and Medicine, Kohnodai Hospital.

\section{Financial Disclosure}

Authors have no financial disclosure to report.

\section{Conflict of Interest}

The authors declare that they have no conflict of interest concerning this article.

\section{Informed Consent}

Not applicable.

\section{Author Contributions}

HY designed the research. SM collected data. HY analyzed data, and wrote the paper. Both authors read and approved the final paper.

\section{References}

1. Fulop M, Eder HA. Plasma triglycerides and cholesterol in diabetic ketosis. Arch Intern Med. 1989;149(9):19972002.

2. Nikkila EA, Huttunen JK, Ehnholm C. Postheparin plasma lipoprotein lipase and hepatic lipase in diabetes mellitus. Relationship to plasma triglyceride metabolism. Diabetes. 1977;26(1):11-21.

3. Nikkila EA, Taskinen MR, Kekki M. Relation of plasma high-density lipoprotein cholesterol to lipoprotein-lipase activity in adipose tissue and skeletal muscle of man. Atherosclerosis. 1978;29(4):497-501. 